\title{
A influência da idade e da escolaridade sobre a experiência empática de adultos
}

\section{Influence of age and schooling degree on adults' empathic experience}

\author{
Vanessa Dordron de Pinho* \\ Universidade do Estado do Rio de Janeiro - UERJ, Rio de Janeiro, RJ, Brasil \\ Conceição Santos Fernandes** \\ Universidade do Estado do Rio de Janeiro - UERJ, Rio de Janeiro, RJ, Brasil \\ Eliane Mary de Oliveira Falcone*** \\ Universidade do Estado do Rio de Janeiro - UERJ, Rio de Janeiro, RJ, Brasil
}

\begin{abstract}
RESUMO
Atualmente, os teóricos começam a compartilhar uma visão da experiência empática humana como um fenômeno multidimensional influenciado por diversos aspectos, dentre eles, a idade e a escolaridade. No presente estudo, investigou-se as relações entre a habilidade social empática e essas duas variáveis sociodemográficas, em 537 participantes adultos. A habilidade empática foi estudada em quatro dimensões, que correspondem aos quatro fatores que compõem o instrumento utilizado, o Inventário de Empatia (IE): Tomada de Perspectiva (TP), Flexibilidade Interpessoal (FI), Altruísmo (Al) e Sensibilidade Afetiva (SA). Os resultados indicaram correlações significativas do ponto de vista estatístico entre idade e dois aspectos da empatia: altruísmo e sensibilidade afetiva. Os grupos de escolaridade se diferenciaram quanto à flexibilidade interpessoal e ao altruísmo. Através deste artigo é possível hipotetizar que as variáveis estudadas estão relacionadas à empatia, corroborando estudos anteriores que apontam como variáveis cognitivas caminham juntas com o desenvolvimento e podem ser afetadas pelo aprimoramento educacional.
\end{abstract}

Palavras-Chave: Empatia, Idade, Grau de escolaridade.

\begin{abstract}
Currently, theorists start sharing a view of human empathic experience like a multidimensional phenomenon influenced by several aspects, among them age and scholarity level. This study aims to investigate relations between empathic social skill and these two sociodemographic variables. The sample was composed by 537 adult participants. The empathic skill was studied across four dimentions of Inventário de Empatia (I.E.): Perspective Taking, Interpersonal Flexibility, Altruism, and Affective Sensibility. The findings have pointed statistically significant correlations between age and some aspects of empathy: altruism and affective sensibility. The scholarity groups are diferentiated by interpersonal flexibility and altruism. Through this paper it's possible to suggest that the studied variables are related to empathy, corroborating previous studies that point out cognitive variables follow
\end{abstract}


together with development and must be affected by educational improvement.

Keywords: Empathy, Age, Scholarity level.

\section{I ntrodução}

Os altos índices de violência que marcam a vida contemporânea têm despertado o interesse de pesquisadores de diversas áreas para o estudo de fatores relacionados tanto a comportamentos antissociais quanto a comportamentos prossociais. Existem fortes evidências de que a habilidade empática e outros conceitos similares, tais como altruísmo e raciocínio moral, funcionam como mediadores da prossociabilidade humana (FALCONE, 2009; HAUSER, 2006).

Diversas pesquisas têm revelado a relevância deste fenômeno na qualidade das relações sociais (FALCONE, 2001; FALCONE; GIL; FERREIRA, 2007; MARQUES, 1999). Pavarino, Del Prette e Del Prette (2005), por exemplo, estudaram a literatura nacional e internacional a fim de identificar as relações entre empatia e agressividade. Os resultados desta revisão apontaram para uma correlação inversa entre essas duas variáveis, sugerindo que déficits em empatia constituem um fator de risco para comportamentos antissociais, especialmente os agressivos.

A revisão da literatura psicológica indica que existem discordâncias acerca da compreensão do fenômeno empático, com alguns autores concebendo a empatia como um fenômeno cognitivo e outros como um fenômeno afetivo (ver STRAYER, 1992). No entanto, com o aumento do conhecimento acerca da biologia do cérebro, evidências começaram a surgir de que a empatia diz respeito a um fenômeno multidimensional.

A partir de estudos neurobiológicos - com neuroimagens e com pacientes com lesões cerebrais -, Decety e Jackson (2004) e Preston e de Waal (2002) identificaram que as bases neurais da empatia estão amplamente distribuídas no cérebro humano, sendo que, formas mais simples de empatia, como o contágio emocional e o malestar pessoal, estão associadas a áreas corticais somatossensoriais e aos sistemas autonômo e límbico, ao passo que as formas mais sofisticadas de empatia, como a empatia cognitiva controlada, dependem de seções diferentes do córtex pré-frontal, além de outras áreas corticais. Isto evidencia a empatia como um fenômeno multidimensional que envolve áreas cerebrais relacionadas às experiências afetivas e áreas cerebrais relacionadas à capacidade cognitiva.

Estudos têm apontado a presença da empatia em diversas espécies animais, porém não com a complexidade encontrada na espécie humana, que por conta do refinamento do córtex pré-frontal alcançou um desempenho mais sofisticado desta habilidade, como por 
exemplo, a acuidade na tomada de perspectiva de outros (DE WAAL, 1996). Desse modo, a empatia humana enquanto uma habilidade social muldimensional pode ser considerada como:

\begin{abstract}
A capacidade de compreender, de forma acurada, bem como de compartilhar ou considerar sentimentos, necessidades e perspectivas de alguém, expressando este entendimento de tal maneira que a outra pessoa se sinta compreendida e validada (FALCONE et al., 2008, p. 323).
\end{abstract}

O componente cognitivo da empatia refere-se a uma capacidade de compreender acuradamente a perspectiva e os sentimentos dos outros (ICKES, 1997) e o componente afetivo refere-se a sentimentos de compaixão/preocupação para com a outra pessoa (FALCONE et al., 2008). Falcone (1999; 2003) aponta ainda para um terceiro componente da empatia, o comportamental, que é entendido como manifestações verbais e não verbais de compreensão dos estados internos da outra pessoa (para uma compreensão mais detalhada do assunto, ver: BARRETT-LENNARD, 1993; FESCHBACH, 1992; GREENBERG; ELLIOTT, 1997).

A literatura sobre o tema da empatia também apresenta estudos sobre fatores que contribuem para o desenvolvimento desta habilidade. Entre estes fatores, encontram-se: o estilo de apego (BRITTON; FUENDELING, 2005; MARK; IJZENDOORN; BAKERMANSKRANENBURG, 2002; PONTES; SILVA; GAROTTI; MAGALHÃES, 2007), as práticas parentais e o oferecimento de modelos pelos pais e cuidadores (GARCIA-SERPA; DEL PRETTE; DEL PRETTE, 2006; MOTTA; FALCONE; CLARK; MANHÃES, 2006), fatores inatos, saúde mental e bases neurocognitivas (SHAMAY-TSOORY; SHUR; HARARI; LEVKOVITZ, 2007) e a idade e a escolaridade (BAILEY; HENRY, 2008; BAILEY; HENRY; VON HIPPEL, 2008; BRYANT, 1982; EISENBERG; CARLO; MURPHY; VAN COURT, 1995; GRÜNH; DIEHL; REBUCAL; LUMLEY; LABOUVIE-VIEF, 2008; TRUSTY; NG; WATTS, 2005). Apenas um estudo foi encontrado avaliando nível socioeconômico, sendo este realizado com uma amostra de crianças (BANDEIRA; ROCHA; FREITAS; DEL PRETTE; DEL PRETTE, 2006), no qual foram encontradas correlações negativas entre comportamentos problemáticos e nível socioeconômico dos pais. Porém as relações com a medida de habilidades sociais não foram verificadas. Em relação, especificamente, à empatia, não foram encontrados estudos avaliando suas relações com o nível socioeconômico.

Bryant (1982) encontrou diferenças significativas em função da idade, ao investigar a empatia entre crianças e adolescentes de diferentes séries, porém não houve controle do efeito de uma variável sobre outra, ou seja, efeito da idade sobre escolaridade e vice-versa. O estudo de Bandeira et al. (2006) mostra, a partir de sua revisão da literatura, uma relação consistente entre o avançar da 
idade, o desempenho acadêmico, problemas de comportamento e, por consequência, a adaptação social, em crianças brasileiras. Além disso, realizaram um estudo com 257 crianças e obtiveram uma correlação significativa entre a idade e o escore global de habilidades sociais, dentre elas a empatia.

Bayley e Henry (2008) encontraram que o componente cognitivo da empatia reduz com o envelhecimento. Isto estaria relacionado com a redução da tomada de perspectiva e teoria da mente, avaliadas a partir de tarefas cognitivas que envolvem a inibição da própria perspectiva. Bailey et al. (2008) encontraram resultados parecidos mostrando que a empatia cognitiva estava mais reduzida em idosos do que em adultos jovens, não encontrando diferenças na empatia afetiva. Trusty, Ng e Watts (2005) encontraram correlações fracas e não significativas entre idade e empatia emocional.

Grünh et al. (2008) realizaram uma pesquisa longitudinal com 400 participantes, quantidade esta que variou ao longo do estudo. $O$ objetivo era investigar se a idade predizia mudanças na empatia autorrelatada. A idade variou entre 10 e 87 anos. Os participantes foram acompanhados por 12 anos e foram avaliados em quatro momentos diferentes. Os autores também avaliaram a relação entre empatia e escolaridade, comparando três grupos: (1) ensino médio; (2) graduação e (3) além do bacharelado. Em um terceiro momento, controlaram a escolaridade para avaliar a relação entre idade e empatia.

O estudo longitudinal não indicou diferenças na empatia em função da idade, mas ao realizarem um cruzamento-seccional com a mesma amostra, foi encontrado que pessoas com idade mais avançada pontuaram mais baixo em empatia. Quanto ao nível de escolaridade, foi indicado que maiores níveis educacionais apresentaram maiores níveis de empatia, isto é, o grupo com formação além do bacharelado apresentou diferenças significativas em comparação aos demais grupos de escolaridade, nas quatro fases do estudo. Com o controle da escolaridade, o padrão de resultados não foi alterado no que diz respeito às relações entre idade e empatia.

É possível pensar também que a própria experiência acadêmica seja um fator facilitador do aprimoramento da capacidade empática. Godoy, Santos e Moura (2001) realizaram um estudo exploratório sobre o impacto dos anos de graduação entre estudantes que estavam terminando o curso superior, a fim de validar um instrumento para avaliar os efeitos da graduação. O questionário desenvolvido parte da premissa que os alunos de graduação passam por um conjunto de experiências curriculares e extracurriculares que proporcionam alterações em suas características cognitivas e/ou afetivas, considerando que as mudanças ocorrem tanto em consequência da maturação física, quanto da influencia ambiental. Eles estavam interessados nas mudanças que se devem à experiência 
do aluno no ensino superior, pois como o estudo foi feito com adulto, a maturação física já havia sido estabilizada.

As respostas indicadas pelos alunos apontaram que a experiência acadêmica influenciou as habilidades pessoais, a preparação para a profissão, a complexidade cognitiva, as habilidades de relacionamento e autoconhecimento, a independência intelectual e a consciência da vida profissional. Assim, suspeita-se que a graduação também exerça influência sobre a habilidade empática.

Pitta, Santos, Escher e Bariani (2000) avaliaram a associação entre a iniciação científica e o estilo cognitivo entre universitárias. As bolsistas de iniciação científica apresentaram, predominantemente, um estilo caracterizado por reflexividade (que diz respeito a pensamentos mais ordenados e contínuos e às ponderações e organizações que antecedem a resposta de uma pessoa) e por divergência de pensamento (que diz respeito à imaginação, criatividade, originalidade e fluência das respostas), o que levou as autoras a concluir que a iniciação científica é um dos fatores que interfere no desenvolvimento cognitivo de estudantes de nível superior. A partir desses resultados, sugere-se que a vivência acadêmica exerça um impacto sobre a empatia, possivelmente em um nível mais cognitivo.

A revisão desses estudos enfatiza a relevância da idade e sugere a influência da escolaridade na experiência empática, uma vez que são variáveis importantes relacionadas ao desenvolvimento cognitivo, que por sua vez, possibilita o desenvolvimento de pensamentos complexos, apontados na literatura como preditores da flexibilidade cognitiva, importante mediadora da flexibilidade interpessoal e da tomada da perspectiva do outro (DECETY; JACKSON, 2004; PRESTON; DE WAAL, 2002). Deste modo, cogita-se a possibilidade de haver uma relação positiva entre a habilidade empática e o grau de escolarização.

A carência de estudos brasileiros que avaliem a habilidade empática entre faixas etárias adultas e que investiguem a influência da vida universitária sobre a experiência empática justificam o presente estudo. Deste modo, a presente pesquisa buscou responder às seguintes perguntas: 1) a empatia se modifica em função da idade entre indivíduos adultos?; 2) pessoas de três diferentes grupos de escolaridade (médio completo, superior incompleto e superior completo) se diferenciam quanto à empatia?; 3) qual o efeito das variáveis quando a idade é controlada?

\section{Método}

\section{Participantes}

A amostra caracteriza-se como não-probabilística acidental, definida por Seidl de Moura e Ferreira (2005) como aquela que utiliza pessoas que se dispõem voluntariamente a colaborar no estudo, respondendo 
aos instrumentos de coleta de dados propostos pelo pesquisador. 0 número de participantes foi de 537, de ambos os sexos, com idades variando de 18 a 79 anos, com média de 32 anos. Dentre os participantes, 100 tinham ensino médio completo, 212 estavam cursando o nível superior e 220 já eram graduados (ver Tabela 1).

Tabela 1 - Dados sociodemográ ficos da amostra

\begin{tabular}{llll}
\hline & $\mathrm{N}$ & Media da idade & Desvio Padrão \\
\hline Amostra Total & 534 & 32,1 & 12,7 \\
\hline $\begin{array}{l}\text { Ensino Médio } \\
\text { Completo }\end{array}$ & 100 & 36,5 & 16,0 \\
\hline $\begin{array}{l}\text { Ensino Superior } \\
\text { Incompleto }\end{array}$ & 212 & 25,8 & 8,6 \\
\hline $\begin{array}{l}\text { Ensino Superior } \\
\text { Completo ou } \\
\text { Acima }\end{array}$ & 220 & 36,1 & 11,8 \\
\hline
\end{tabular}

\section{Materiais e I nstrumentos de avaliação}

Foi utilizada uma ficha para a obtenção de dados pessoais da amostra, contendo perguntas relativas à idade, gênero, profissão e grau de instrução de cada participante e foi aplicado um inventário de autorrelato para avaliar a habilidade da empatia - Inventário de Empatia - IE (FALCONE et al., 2008). O instrumento é composto por 40 itens, alocados em quatro fatores, que avaliam os aspectos cognitivo, afetivo e comportamental da empatia. Os fatores são: Fator 1, Tomada de perspectiva (TP), caracterizado por uma capacidade de entender a perspectiva e sentimentos da outra pessoa, principalmente em situações nas quais ocorre conflito de interesses. Esse fator é composto por 12 itens, com consistência interna (Alpha de Cronbach) de 0,85. O fator 2, Flexibilidade interpessoal (FI), caracteriza-se por uma capacidade em aceitar perspectivas, crenças e valores muito diferentes, além de tolerância à frustração provocada pela atitude do interlocutor; pessoas com elevado nível em FI tendem a fazer menos julgamentos e críticas e sabem regular as próprias emoções. Esse fator está composto por 10 itens e seu Alpha foi de 0,77 . O fator 3 , denominado Altruísmo (Al), caracteriza-se por uma capacidade de sacrificar temporariamente as próprias necessidades, em benefício de outra pessoa; indivíduos com escore baixo em Al tendem a reagir de forma egoísta, demonstrando pouco interesse pelas necessidades das outras pessoas. O Al apresenta 9 itens e Alpha de 0,75. Por último, o fator 4, nomeado de Sensibilidade afetiva (SA), representa a tendência a experimentar sentimentos de compaixão e de interesse pelo estado emocional de outra pessoa, denotando preocupação genuína com o bem-estar dos outros e um cuidado com os efeitos do próprio comportamento sobre o bem-estar do outro; apresenta 9 itens e Alpha de 0,72. 


\section{Procedimentos para coleta de dados}

O estudo teve início após aprovação pelo Comitê de Ética em Pesquisa da Universidade do Estado do Rio de Janeiro - COEP/UERJ . A aplicação da escala foi feita individualmente, em uma amostra de conveniência. O critério de exclusão foi ter idade a partir de 18 anos e escolaridade a partir do ensino médio completo. Os participantes tinham a opção de responder o inventário no momento do encontro com o pesquisador ou levá-lo para responder em casa e devolvê-lo em uma data combinada previamente.

Os objetivos da pesquisa eram explicitados através do Termo de Consentimento Livre e Esclarecido, sendo garantido aos participantes que sua participação seria voluntária. Foi enfatizado, ainda, que o anonimato dos mesmos seria preservado. Não foi estabelecido um limite de tempo para responder à escala. Após a devolução dos instrumentos, eles eram revisados para verificar se todos os itens haviam sido respondidos.

\section{Análises dos Dados}

Para investigar as relações entre a idade e as quatro dimensões do $I E$, foi realizada a análise de correlação de Pearson. Uma análise Anova foi conduzida para verificar as possíveis diferenças entre os três grupos de escolaridade quanto aos quatro fatores da empatia. Por fim, foi realizada a análise Ancova, para verificar o padrão de resultados, após o controle da idade.

\section{Resultados}

\section{Efeito da I dade}

A partir da análise de Correlação de Pearson (ver Tabela 2), encontrou-se uma correlação significativa e negativa entre idade e Altruísmo ( $r=-0,17 ; p<0,001)$, e uma correlação significativa e positiva entre idade e Sensibilidade Afetiva $(r=0,09 ; p<0,05)$, indicando que quanto maior a idade, menor o altruísmo e que quanto maior a idade, maior a sensibilidade afetiva.

\section{Tabela 2 - Correlação de Pearson Idade $x \mathbb{E}$}

\begin{tabular}{lllll}
\hline Idade & TP & FI & A1 & SA \\
\hline $\mathrm{N}$ & 529 & 534 & 534 & 532 \\
\hline$r$ & 0,01 & 0,08 & $-0,17^{* *}$ & $0,09 *$ \\
\hline $\mathrm{P}$ & 0,77 & 0,06 & 0,00 & 0,03 \\
\hline $\mathrm{p}_{\mathrm{p} 001} 0,05$ & & & &
\end{tabular}




\section{Efeito da Escolaridade}

Os testes da ANOVA que foram realizados para cada uma das quatro subescalas da empatia (ver Tabela 3 ) mostraram que os grupos de escolaridade se diferiam quanto ao Altruísmo $(F=9,29$ e $p<0,001)$ e à Flexibilidade Interpessoal ( $F=5,06$ e $p<0,01)$.

\section{Tabela 3 - Anova Escolaridade x IE}

\begin{tabular}{lll}
\hline $\mathbb{I E}$ & $\mathrm{F}$ & $\mathrm{P}$ \\
\hline $\mathrm{IP}$ & 0,72 & 0,49 \\
\hline FI & $5,06^{*}$ & 0,01 \\
\hline $\mathrm{A} 1$ & $9,29 * *$ & 0,00 \\
\hline $\mathrm{SA}$ & 0,28 & 0,76 \\
\hline${ }^{*} \mathrm{p}, 0,01$ & &
\end{tabular}

O teste post hoc de Bonferroni foi realizado em seguida para verificar quais grupos de escolaridade se diferiam quanto ao $\mathrm{Al}$ e à $\mathrm{FI}$. Observaram-se as seguintes diferenças entre os grupos:

- para o fator Al, o grupo de escolaridade média completa se diferenciou estatisticamente de modo significativo dos grupos de escolaridade superior incompleta $(p<0,001)$ e de escolaridade superior completa $(p<0,05)$.

- para o fator $\mathrm{FI}$, o grupo de escolaridade média completa se diferenciou estatisticamente de modo significativo dos grupos de escolaridade superior incompleta $(p<0,05)$ e de escolaridade superior completa $(p<0,01)$.

Em ambos os fatores, Al e FI, pessoas com nível superior (incompleto ou completo) apresentaram maiores escores que pessoas que têm apenas o nível médio completo.

\section{Análise Ancova}

Uma análise Ancova foi feita, utilizando a variável idade como covariável, a fim de verificar os efeitos da escolaridade sobre as dimensões do I.E. sem a possível influência da idade sobre os fatores. Os resultados obtidos (ver Tabela 4) mostraram que o efeito da escolaridade sobre a Flexibilidade Interpessoal $(F=6.0 ; p<0.001)$ e sobre o Altruísmo $(\mathrm{F}=5.4 ; \mathrm{p}<0.01)$ se manteve, mesmo após o controle da variável idade. 
Tabela 4 - Ancova com controle da idade

\begin{tabular}{lrll}
\hline IE & & $\mathrm{F}$ & $\mathrm{p}$ \\
\hline IP & Idade & 0,32 & 0,60 \\
& Escolaridade & 0,83 & 0,44 \\
\hline FI & Idade & 5,00 & 0,03 \\
& Escolaridade & $6,00^{* *}$ & 0,00 \\
\hline A1 & Idade & 7,53 & 0,01 \\
& Escolaridade & $5,40^{*}$ & 0,01 \\
\hline SA & Idade & 5,26 & 0,02 \\
& Escolaridade & 0,40 & 0,67 \\
\hline$* *$ p $<0,001$ & & &
\end{tabular}

Pode-se observar que, ao se controlar a idade, o efeito da escolaridade sobre a Flexibilidade Interpessoal aumentou (passando de $F=5.06$ para $F=6.00$ ), mostrando que parte do efeito inicial atribuído à idade se devia, na verdade, à escolaridade. Já no caso do Altruísmo, o efeito da escolaridade diminuiu (passando de $\mathrm{F}=9.29$ para $\mathrm{F}=5.40$ ), indicando que parte do efeito inicial atribuído à escolaridade, se devia à idade.

\section{Discussão}

Os resultados do presente estudo apontaram diferenças em alguns aspectos da empatia em relação à idade e à escolaridade. Observouse que, quanto maior a idade, menor o altruísmo e maior a sensibilidade afetiva, e que, quanto maior escolaridade, maior os níveis de flexibilidade interpessoal e de altruísmo.

Sobre a idade

Os níveis de altruísmo decaíram com o aumento da idade, embora os níveis de sensibilidade afetiva fossem maiores em sujeitos mais velhos. Assim, parece que quanto maior a idade menor a capacidade de abrir mão das próprias necessidades, característica representada pelo fator Altruísmo, mas, por outro lado, aumenta-se a possibilidade de sentir interesse e preocupação pelo outro, medido através do fator Sensibilidade Afetiva.

Por definição, o altruísmo acarreta um custo inicial para aquele que é altruísta, e conseqüências positivas podem vir a ocorrer somente após um tempo significativo. Além disso, a doação excessiva ao outro pode acarretar mais prejuízos para si e para a relação interpessoal que benefícios. Também é possível que indivíduos excessivamente altruístas estejam motivados por um esquema disfuncional de auto sacrifício ou subjugação (YOUNG, 2003).

Esses resultados podem, então, indicar que a experiência adquirida com os anos de vida favoreça o aumento da capacidade de se voltar 
afetivamente para o outro e, ao mesmo tempo, ser importante na modulação do comportamento altruísta, de modo que este não se torne disfuncional, podendo transformar-se em auto sacrifício. É possível, assim, que essa moderação do altruísmo seja uma estratégia que equilibra esse comportamento de abrir mão das próprias necessidades em prol do outro, não significando que essas pessoas não sejam altruístas, contudo elas passam a selecionar melhor em que momento essa atitude trará benefícios para os envolvidos.

A sensibilidade afetiva se refere a uma tendência a considerar as necessidades do outro. Essa característica tem sido considerada como relevante para uma boa convivência e, portanto, adaptativa, pois proporciona maior vínculo com outras pessoas. Entretanto, o fato do individuo aumentar sua capacidade de sensibilidade afetiva não significa que necessariamente vá aumentar seus níveis de altruísmo, porque este, como discutido anteriormente, pode ser moderado por fatores cognitivos. Como há esta modulação, ele não ocorrerá sempre de forma incondicional ou vinculada à capacidade de sensibilidade afetiva, ou seja, de considerar o estado interno do outro.

O estudo não revela diferenças significativas quanto a aspectos cognitivos da empatia (Tomada de Perspectiva e Flexibilidade Interpessoal), o que não foi observado em estudos anteriores, nos quais o aprimoramento do aspecto cognitivo ocorria até a fase de adolescência (BANDEIRA et al., 2006), e seu declínio no envelhecimento (BAYLEY; HENRY, 2008).

$\mathrm{Na}$ infância e adolescência, várias destrezas cognitivas, dentre elas a capacidade de perceber sinais de outras pessoas, reconhecer frases enganosas, etc., aumentam com a idade. Eisenberg, Murphy e Shepard (1997) apontaram que as crianças são capazes de reconhecer a comunicação verbal enganosa, ou seja, quando as pessoas simulam ou tentam esconder emoções, já no período de 9 e 11 anos, entretanto ainda não são capazes de inferir acuradamente os pensamentos e sentimentos de outras pessoas em situações enganosas. Por outro lado, alguns estudos realizados no envelhecimento (BAILEY et al., 2008) também apontam um déficit de empatia cognitiva em idosos, correlacionado ao declínio cognitivo de funções de controle inibitório, funções cognitivas relacionadas a funções executivas, dentre elas, a flexibilidade cognitiva, notadamente associada a aspectos da empatia como a tomada de perspectiva (DECETY; JACKSON, 2004; SHAMAY-TSOORY; TOMER; BERGER; AHARON-PERETZ, 2003).

Estas fases de desenvolvimento, tanto a infância e adolescência quanto o envelhecimento, são momentos importantes nos quais ocorrem variações nas funções executivas. O fato de não terem sido encontradas diferenças significativas entre adultos quanto aos aspectos cognitivos da empatia pode indicar que funções cognitivas 
relacionados a esses componentes estejam estáveis na vida adulta, o que também não significa que não possam se desenvolver quando estimuladas.

Sobre a escolaridade

Com relação à escolaridade, foi encontrado que indivíduos com nível superior, completo ou incompleto, apresentaram níveis mais elevados de altruísmo e de flexibilidade interpessoal quando comparados a indivíduos com escolaridade média completa, sugerindo que a escolaridade superior possa desempenhar um importante papel em aspectos afetivos e cognitivos da empatia. Esses resultados são condizentes com os encontrados em estudos anteriores, como, por exemplo, os de Godoy et al. (2001) e os de Pitta et al. (2000), que encontraram que a vivência universitária exerce impacto sobre aspectos sociais, técnicos, cognitivos e afetivos dos estudantes.

Parrat-Dayan (2007) ressalta a importância da atividade discursiva para o desenvolvimento da flexibilidade cognitiva e discute como o confrontamento com diversas opiniões, muitas vezes bastante divergentes entre si, gera no indivíduo a necessidade de entender o ponto de vista do outro, ou pelo menos respeitá-lo, desenvolvendo assim a tolerância para com modos de pensar muito diferentes do seu. Sabendo-se que as discussões em grupo são recorrentes na vida acadêmica, elas podem estar associadas ao melhor desempenho empático em grupos com ensino superior.

Os resultados acerca da influência do ensino superior sobre a experiência empática também podem ser entendidos em função das oportunidades oferecidas pela vida acadêmica. Esse tipo de vivência cria oportunidades diversas para o desenvolvimento moral do indivíduo, bem como para o desenvolvimento de processos cognitivos mais complexos, através de suas atividades curriculares e extracurriculares, como trabalhos em grupo, discussões sobre temas polêmicos e/ou de cunho moral, convivência com distintas pessoas, contato com o modo de pensar científico, aprendizagem de teorias que se vão tornando cada vez mais abstratas e complexas, entre outras.

Os resultados, por outro lado não indicaram relações entre o nível de escolaridade e os fatores Tomada de Perspectiva e Sensibilidade Afetiva, considerados componentes cognitivo e afetivo do inventário de empatia, respectivamente. Cogita-se a possibilidade dessas dimensões da empatia também sofrerem influência do grau de escolaridade, no entanto, essa hipótese pode não ter sido corroborada no presente estudo por uma limitação da amostra, composta por pessoas com um padrão de instrução relativamente alto em relação à população brasileira, devendo a hipótese ser melhor explorada em estudos futuros. 


\section{Controle da variável idade}

O controle da idade não afetou o padrão geral dos resultados, além de permitir a delimitação do efeito das variáveis estudadas sobre os aspectos da empatia. Percebe-se, a partir dessa análise, que mesmo tendo sido realizado um estudo com uma amostra de adultos, que teoricamente teriam alcançado a maturação cognitiva e emocional, os aspectos da empatia podem ser modificados a partir de novos estímulos e aprendizagens. Um exemplo foi o efeito da experiência acadêmica universitária sobre dimensões afetivas e cognitivas da empatia (Flexibilidade interpessoal e Altruísmo).

\section{Conclusões}

No presente estudo, utilizou-se um instrumento que contempla diferentes dimensões da empatia, uma vez que esta habilidade tem sido descrita na literatura como uma variável multidimensional. A escolha pelo IE foi positiva porque permitiu investigar diferenças entre grupos quanto a aspectos afetivos e cognitivos da habilidade empática, mostrando que as variáveis independentes podem estar relacionadas a alguns aspectos da experiência da empatia, mas não a outros.

Entretanto, o estudo apresentou algumas limitações, como o fato de não ter contemplado um número significativo de participantes idosos, - que pode ter contribuído para que alguns resultados não tenham sido encontrados, corroborando estudos anteriores. Estudos futuros podem ser conduzidos nesse sentido, incluindo faixas etárias a partir dos 60 anos de idade, para avaliar características mais particulares de cada faixa etária.

Além disso, não foram realizadas avaliações com níveis baixos de escolaridade, o que poderia ter apontado diferenças em outros fatores da empatia, como a Tomada de Perspectiva. Além dos grupos de escolaridade estudados, grupos formados por pessoas com 0 ensino fundamental, completo e incompleto, precisam ser incluídos em estudos posteriores. Seria interessante investigar ainda, se o tipo de universidade (por exemplo, se oferece ou não acesso à experiência de iniciação científica, se estimula ou não a participação em congressos, se o curso é presencial ou à distância), o turno em que se estuda (se de manhã, à tarde, à noite ou integral), a área cursada (ciências humanas, sociais, exatas, biomédicas ou tecnológicas) etc. influem na experiência empática.

É preciso destacar, ainda, que este estudo não permite ver a evolução da empatia com o passar dos anos, por trabalhar com indivíduos de diferentes grupos, e não com os mesmos indivíduos ao longo do tempo. Desse modo, estudos longitudinais futuros são relevantes para acompanhar e detectar com mais precisão as mudanças ocorridas nessa habilidade com o decorrer dos anos. Contudo, como pesquisas sobre mudanças na habilidade empática 
durante a vida adulta e o ensino superior são escassas, o presente estudo trouxe uma contribuição aos anteriores, na medida em que avaliou diferentes níveis de empatia em uma ampla faixa etária de indivíduos adultos e em diferentes graus de escolaridade, incluindo o nível superior, abrindo um novo leque para investigações futuras.

\section{Referências Bibliográficas}

BAILEY, P. E.; HENRY, J. D. Growing Less Empathic With Age: Disinhibition of the Self-Perspective. Journal of Gerontology, Sydney, v. 63, n. 4, p. 219-226, jul. 2008.

BAILEY, P. E., HENRY, J. D.; VON HIPPEL, W. Empathy and social functioning in late adulthood. Aging and Mental Health, Sydney, v. 12, n. 4, p. 499-503, jul. 2008.

BANDEIRA, M.; ROCHA, S. S.; SOUZA, T. M. P.; DEL PRETTE, Z. A. P.; DEL PRETTE, A. Comportamentos problemáticos em estudantes do ensino fundamental: características da ocorrência e relação com habilidades sociais e dificuldades de aprendizagem. Estudos de Psicologia, Natal, v. 11, n. 2, p. 199-208, mai./ago. 2006.

BARRETT-LENNARD, G. T.The phases and focus of empathy. The British Psychological Society, Leicester, v. 66, n. 1, p. 3-14, mar. 1993.

BRITTON, P. C.; FUENDELING, J. M. The Relations Among Varieties of Adult Attachment and the Components of Empathy. The Journal of Social Psychology, Washington, v. 145, n. 5, p. 519-530, out. 2005.

BRYANT, B. K. An index of empathy for children and adolescents. Child Development, New Orleans, v. 53, n. 2, p. 413- 425, abr. 1982.

DECETY, J.; JACKSON, P. L. The functional architecture of human empathy. Behavioral and Cognitive Neuroscience Reviews, Indiana, v. 3, n. 2, p. 71-100, jun. 2004.

DE WAAL, F. Good Natured: the origins of right and wrong in humans and other animals. Cambridg; London: Harvard University Press, 1996.

EISENBERG, N.; CARLO, G.; MURPHY, B.; VAN COURT, P. Prosocial development in late adolescence: a longitudinal study. Child Development, New Orleans, v. 66, n. 4, p. 1179-1197, ago. 1995. EISENBERG, N.; MURPHY, B. C.; SHEPARD, S. The development of empathic accuracy. In: ICKES, W. (Ed.). Empathic accuracy. New York: Guilford, 1997.

FALCONE, E. M. O. A avaliação de um programa de treinamento da empatia com universitários. Revista Brasileira de Terapia Comportamental e Cognitiva, São Paulo, v. 1, n.1, p. 23-32, jun. 1999. 
FALCONE, E. M. O. Uma proposta de um sistema de classificação das habilidades sociais. In: GUI LHARDI, H. J.; MADI, M. P.; QUEIROZ, P. P.; SCOZ, M. C. (Orgs.). Sobre comportamento e cognição: expondo a variabilidade. Santo André: ESETEC, 2001.

FALCONE, E. M. O. Empatia. In: ABREU, C. N.; ROSO, M. (Orgs.). Psicoterapias cognitiva e construtivista. Novas fronteiras da prática clínica. Porto Alegre: Artmed, 2003.

FALCONE, E.; FERREIRA, M. C., LUZ, R. C. M, FERNANDES, C. S.; FARIA, C. A.; D’ÀUGUSTIN, J.; SARDINHA, A.; PINHO, V. D. Inventário de Empatia (I.E.): Desenvolvimento e Validação de uma medida brasileira. Avaliação Psicológica, Porto Alegre, v. 7, n. 3, p. 321-334, dez. 2008.

FALCONE, E.; GIL, D. B.; FERREIRA, M. C. Um estudo comparativo da freqüência de verbalização empática entre psicoterapeutas de diferentes abordagens teóricas. Estudos de Psicologia, Campinas, v. 24, n. 4, p. 451-461, out./dez. 2007.

FALCONE, E. M. O. Empatia: a sabedoria do vínculo afetivo e das relações sociais. In: II SEMINÁRIO INTERNACIONAL DE HABILIDADES SOCIAIS, 2009, Rio de Janeiro. Anais do II Seminário I nternacional de Habilidades Sociais, Rio de Janeiro, UERJ , 2009. p. 5-14.

FESHBACH, N. D. Empatia parental y ajuste infantil. In: EISENBERG, N.; STRAYER, J. (Orgs.). La empatía y su desarrollo. Bilbao: Desclée de Brower, 1992.

GARCIA-SERPA, F. A.; DEL PRETTE, Z. A. P; DEL PRETTE, A. Meninos Pré-Escolares Empáticos e Não-Empáticos: Empatia e Procedimentos Educativos dos Pais. Revista Interamericana de Psicología/ Interamerican Journal of Psychology, Porto Alegre, v. 40, n. 1, p. 77-88, abr. 2006.

GODOY, A. S.; SANTOS, F. C.; MOURA, J. A. Avaliação do impacto dos anos de graduação sobre os alunos. Estudo exploratório com estudantes do último dos cursos de Ciências Contábeis e Administração de uma faculdade particular de São Paulo. Revista Administração on line [On Line]. FECAP, São Paulo, v. 2, n. 1, jan./fev./mar. 2001. Disponível em: <http://www.fecap.br/adm_online/>. Acesso em: 19 mar. 2009.

GREENBERG, L. S.; ELLIOTT, R. Varieties of empathic responding. In: BOHART, A. C.; GREENBERG, L. S. (Eds.). Empathy reconsidered. New directions in psychotherapy. Washington - DC: American Psychological Association, 1997.

GRÜHN, D.; REBUCAL, K.; DIEHL, M.; LUMLEY, M.; LABOUVIE-VIEF, G. Empathy across the adult lifespan: longitudinal and experiencesampling findings. Emotion, Washington, v. 8, n. 6, p. 753-765, dez. 2008.

HAUSER, M. D. Moral Minds: The nature of right and wrong. New York: Harper Perennial, 2006. 
ICKES, W. Introduction. In: ICKES, W. (Ed.). Empathic accuracy. New York: Guilford, 1997.

MARK, I. L.; IJZENDOORN, M. H.; BAKERMANS-KRANENBURG, M. J. Development of empathy in girls during the second year of the life: associations with parenting, attachment, and temperament. Social Development, v. 11, n. 4, p. 451-468, nov. 2002.

MARQUES, A. L. Competência social, empatia e representação mental da relação de apego em famílias em situação de risco. 1999. Dissertação (Mestrado em Psicologia do Desenvolvimento) Programa de Pós-graduação em Psicologia do Desenvolvimento, Instituto de Psicologia, Universidade Federal do Rio Grande do Sul, Rio Grande do Sul.

MOTA, D. C.; FALCONE, E.; CLARK, C.; MANhAES, A. C. Práticas educativas positivas favorecem o desenvolvimento da empatia em crianças. Psicologia em Estudo, Maringá, v. 11, n. 3, p. 523-532, set./dez. 2006.

PARRAT-DAYAN, S. A discussão como ferramenta para o processo de socialização e para a construção do pensamento. Educação em Revista, Belo Horizonte, n. 45, p. 13-23, jun. 2007.

PAVARINO, M. G.; DEL PRETTE, A.; DEL PRETTE, Z. A. P. O desenvolvimento da empatia como prevenção da agressividade na infância. Psico, Porto Alegre, v. 36, n. 2, p. 127-134, mai./ago. 2005.

PITTA, K. B.; SANTOS, L. A. D.; ESCHER, C. A.; BARIANI, I. C. D. Estilos cognitivos de estudantes de psicologia: impacto da experiência em iniciação científica. Psicologia Escolar e Educação, Campinas, v. 4, n. 2, 41-49, dez. 2000.

PONTES, F. A. R.; SILVA, S. S. C.; GAROTTI, M.; MAGALHÃES, C. M. C. Teoria do apego: elementos para uma concepção sistêmica da vinculação humana. Aletheia, Canoas, n. 26, p. 67-79, dez. 2007.

PRESTON, S. D.; DE WAAL, F. B. M. Empathy: Its ultimate and proximate Bases. Behavioral and Brain Sciences, Cambridge, v. 25, n. 1, p. 1-72, fev. 2002.

SeIdL De MOURA, M. L.; FerReirA, M. C. Projetos de Pesquisa: elaboração, redação e apresentação. Rio de Janeiro: Eduerj, 2005.

SHAMAY-TSOORY, S. G.; SHUR, S.; HARARI, H.; LEVKOVITZ, Y. Neurocognitive Basis of Impaired Empathy in Schizophrenia. Neuropsychology, Washington, v. 21, n. 4, p. 431-438, jul. 2007. SHAMAY-TSOORY, S. G., TOMER, R., BERGER, B. D.; AHARONPERETZ, J. Characterization of empathy deficits following prefrontal brain damage: The role of the right ventromedial prefrontal cortex. J ournal of Cognitive Neuroscience, Massachusetts, v. 15, n. 3, p. 324-337, abr. 2003.

STRAYER, J. Perspectivas afectivas y cognitivas sobre la empatía. In: EISENBERG, N.; STRAYER, J. (Orgs.). La empatía y su desarrollo. Bilbao: Desclée de Brower, 1992. 
TRUSTY, J.; NG, K.; WATTS, R. Model of Effects of Adult Attachment on Emotional Empathy of Counseling Students. Journal of Counseling \& Development, Michigan, v. 83, n. 1, p. 66-77, jan. 2005.

YOUNG, J. E. Terapia cognitiva para transtornos da personalidade: uma abordagem focada em esquemas. 3 ed. Porto Alegre: Artmed, 2003.

\section{Endereço para correspondência}

Vanessa Dordron de Pinho

Rua São Francisco Xavier, 524, bloco F, $10^{\circ}$ andar, Secretaria do Programa de PósGraduação em Psicologia Social da UERJ, CEP 20550-013, Rio de Janeiro - RJ, Brasil Endereço eletrônico: vanessanessapsi@gmail.com

\section{Conceição Santos Fernandes}

Rua São Francisco Xavier, 524, bloco F, $10^{\circ}$ andar, Secretaria do Programa de PósGraduação em Psicologia Social da UERJ, CEP 20550-013, Rio de Janeiro - RJ, Brasil Endereço eletrônico: conceicaosf@yahoo.com.br

Eliane Mary de Oliveira Falcone

Rua São Francisco Xavier, 524, bloco F, $10^{\circ}$ andar, Secretaria do Programa de PósGraduação em Psicologia Social da UERJ, CEP 20550-013, Rio de Janeiro - RJ, Brasil Endereço eletrônico: elianefalcone@uol.com.br

Recebido em: 19/04/2010

Reformulado em: 06/07/2010

Aceito para publicação em: 07/07/2010

Acompanhamento do processo editorial: Adriana Benevides Soares

\footnotetext{
Notas

* Psicóloga da Fundação de Saúde de Angra dos Reis/RJ; Mestranda do Programa de Pós-Graduação em Psicologia Social da Universidade do Estado do Rio de Janeiro UERJ

**Psicóloga clínica; Mestranda do Programa de Pós-Graduação em Psicologia Social da Universidade do Estado do Rio de Janeiro - UERJ

***Docente do Programa de Pós-Graduação em Psicologia Social da Universidade do Estado do Rio de J aneiro - UERJ
} 